\title{
The rise and fall of basal bodies in the nematode Caenorhabditis elegans
}

\author{
Inna V. Nechipurenko * (1) and Piali Sengupta * (1)
}

\begin{abstract}
The free-living nematode, Caenorhabditis elegans, is a widely used genetic model organism for investigations into centriole and cilia biology. Only sensory neurons are ciliated in C. elegans; morphologically diverse cilia in these neurons are nucleated by basal bodies located at the dendritic endings. C. elegans centrioles comprise a central tube with a symmetric array of nine singlet microtubules. These singlet microtubules remodel in a subset of sensory neurons to form the doublet microtubules of the basal bodies. Following initiation of ciliogenesis, the central tube, but not the outer centriole wall, of the basal body degenerates. Recent ultrastructural characterization of basal body architecture and remodeling have laid the foundation for future studies into mechanisms underlying different aspects of basal body genesis, remodeling, and intracellular positioning.
\end{abstract}

Keywords: C. elegans, Sensory neurons, Centriole, Basal body, Degeneration, Central tube, Diverse cilia morphologies, Ultrastructure

\section{Caenorhabditis elegans: the organism}

The genus Caenorhabditis belongs to the diverse order of Rhabditida that includes free-living as well as parasitic species within the phylum Nematoda and superphylum Ecdysozoa. The model organism, Caenorhabditis elegans, is a free-living bacteriovorous nematode that is found in multiple geographical locations [1]. Although C. elegans was originally identified in compost heaps [2], these nematodes are also found in association with rotting fruits and other decomposing plant material $[1,3-5]$. The commonly used N2 laboratory reference strain was originally isolated in Bristol, England, in 1951 but was passaged for many generations before being frozen [6]. As a consequence, this strain contains multiple fixed alleles that are likely to be adaptive for laboratory growth conditions [6]. Related free-living species that are also studied experimentally include Caenorhabditis briggsae, Caenorhabditis remanei, Caenorhabditis brenneri, and Caenorhabditis japonica $[4,7]$.

*Correspondence: ivn@brandeis.edu; sengupta@brandeis.edu Department of Biology and National Center for Behavioral Genomics, Brandeis University, Waltham, MA 02454, USA

\section{Diverse cilia in C. elegans}

Caenorhabditis elegans populations contain both hermaphrodites and males. 60 ciliated cells are present in common in adult hermaphrodites and males, but males also contain additional ciliated cells [8-12]. All ciliated cells in both males and hermaphrodites are postmitotic sensory neurons, with the majority of male-specific ciliated neurons implicated in the regulation of mating behaviors $[13,14]$. All cilia in $C$. elegans are non-motile and located at the dendritic endings of sensory neurons (Fig. 1a-c). These cilia are remarkably diverse in their appearance and ultrastructure, ranging from simple rod-like structures to cilia exhibiting highly branched, curved, or large fanlike morphologies $[8,9,15,16]$ (Fig. 1b, c). Underlying axonemal structures are also diverse and are built via neuron-specific deployment of tubulin isotypes, intraflagellar transport mechanisms, and ciliary trafficking pathways (e.g. [16-22]). The diversity of cilia structures, and the conservation of many ciliary and centriole/basal body genes in C. elegans, together with the fact that cilia are not essential for its viability, have made this organism an invaluable model for investigations into centriole and cilia biology. 


\section{a}

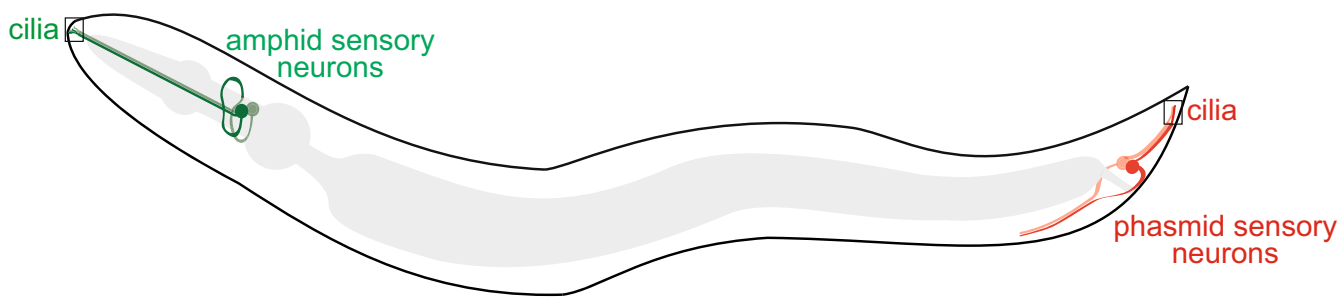

b

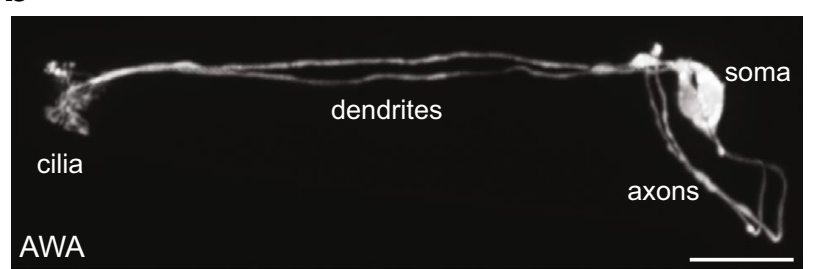

C

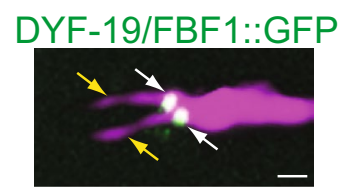

Fig. 1 Ciliated sensory neurons in C. elegans. a Diagram (not to scale) of a C. elegans hermaphrodite showing a subset of the amphid (head) and phasmid (tail) ciliated sensory neurons (green and red cells, respectively). The intestine and pharynx (gray) are also shown. b A confocal image of the amphid AWA sensory neuron pair in an adult hermaphrodite. Anterior is to the left. Note the extensively branched cilia of this neuron type. Image courtesy: Ashish K. Maurya. c A confocal image showing two cilia (yellow arrows) present at the dendritic ending of an amphid AWB neuron in the adult hermaphrodite. The GFP-tagged DYF-19/FBF1 transition fiber protein (white arrows) is localized to the base of the cilia. Anterior is at left. Scale bars in $\mathbf{b}$ and $\mathbf{c} 10$ and $1 \mu \mathrm{m}$, respectively

\section{Basal body origins and structure \\ Centriole structure}

Caenorhabditis elegans centrioles are typically smaller than vertebrate centrioles, and are approximately $\sim 100 \mathrm{~nm}$ in width and $\sim 100-180 \mathrm{~nm}$ in length [23-25]. These centrioles are composed of a radially symmetric array of nine singlet microtubules (sMTs) surrounding a central tube [24-26] (Fig. 2b) which, in a recent study, was shown to contain a cartwheel-like structure similar to that present in centrioles of other organisms [23]. In the early embryo, centriole sMTs appear to be associated with electron-dense 'appendages' [24] (Fig. 2a, b), also termed a 'paddlewheel' [23] of unknown function. At later embryonic stages, hook-like appendages associated with centriole sMTs close to form the B-tubules of the basal body in a subset of ciliated sensory neurons (see below) [25] (Fig. 2c, d).

\section{Centriole to basal body conversion and basal body structure}

56 of the 60 ciliated sensory neurons shared between hermaphrodites and males are born during embryogenesis, whereas all male-specific ciliated neurons, except for the four cephalic CEM neurons, develop post-embryonically $[11,12]$. To date, centriole to basal body conversion has been characterized only in a subset of embryonically generated ciliated sensory neurons of the bilateral amphid organs of the head in the C. elegans hermaphrodite [25, 26] (Fig. 1a).

Caenorhabditis elegans embryogenesis follows a temporally stereotyped progression with an early proliferative stage followed by a later organogenesis/morphogenesis stage [11]. Following the birth of terminally differentiated amphid sensory neurons at the beginning of the organogenesis/morphogenesis period (Fig. 2a) and initial outgrowth of their dendrites, the dendritic tips are anchored in place at the presumptive nose in the embryo, while the neuronal soma translocate posteriorly [27]. Examination of dendritic endings following the commencement of somal translocation showed the presence of a single centriole/basal body, consistent with the presence of a single cilium in the majority of amphid sensory neurons [25, 26]. However, two centrioles/basal bodies were also observed in a subset of neurons, presumably correlating with two cilia found in each of the remaining three amphid sensory neuron pairs $[25,26]$ (see Fig. 1c). The origin of the second basal body in these neurons is unknown.

Visualization of centrioles in a subset of amphid sensory neurons immediately following terminal differentiation showed that the hook-like appendages associated with the A-tubules of the centrioles close to form doublet (AB) MTs (dMTs) of the basal body [25, 26] (Fig. 2c, d). This A- to AB-tubule conversion occurs 


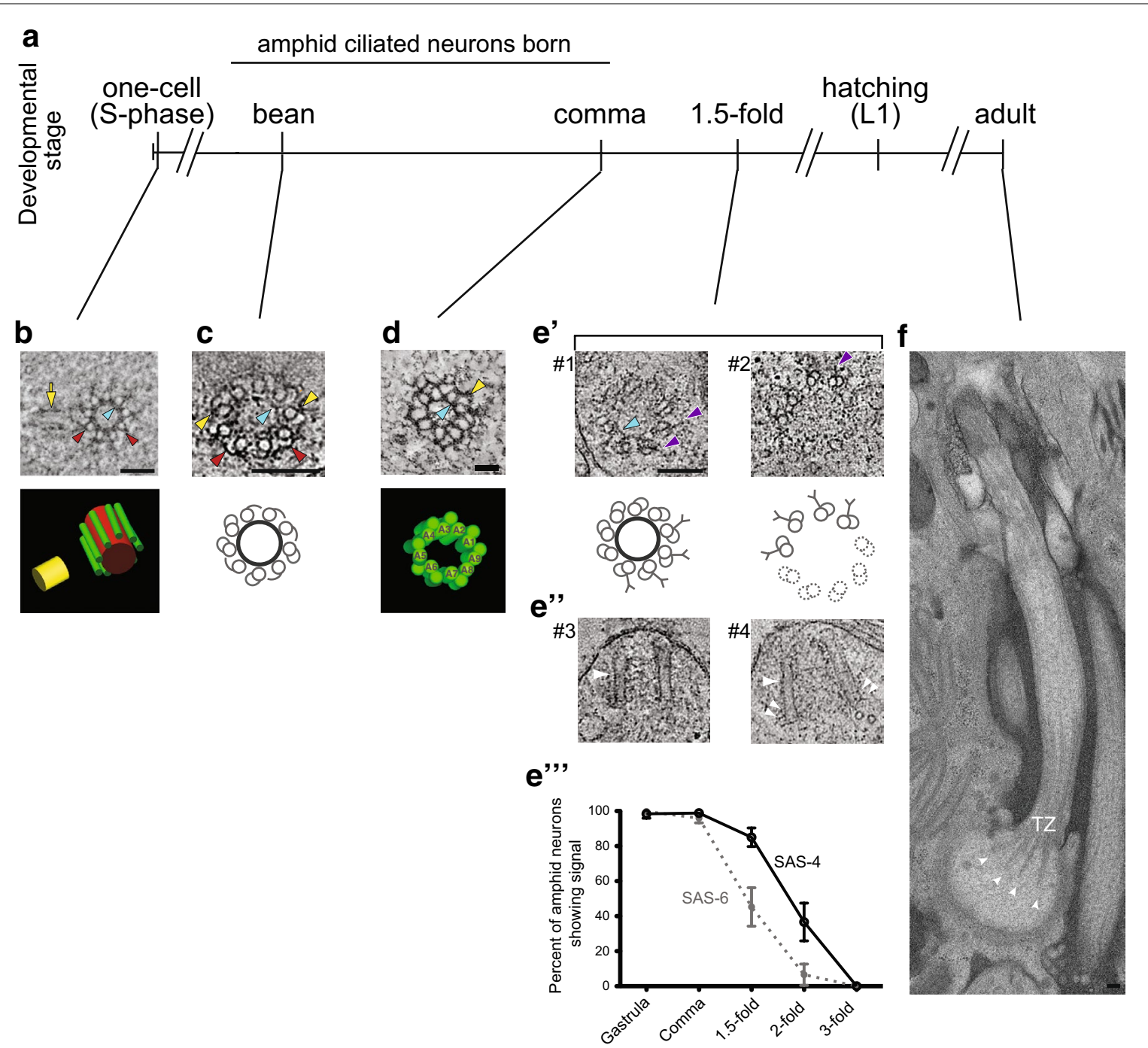

Fig. 2 Ultrastructure of C. elegans centrioles and basal bodies. a Developmental timeline of C. elegans and time of birth of amphid sensory neurons. b-e' $\mathbf{e}^{\prime}$ Representative cross-section tomographic slices and three-dimensional models or cartoons showing centrioles in the one-cell embryo and basal bodies in amphid neurons at the indicated developmental stages. Note basal bodies containing (\#1) or lacking (\#2) a central tube in $\mathbf{e}^{\prime}$. Central tube, sMTs, and the daughter centriole are indicated in red, green and yellow, respectively in the model in $\mathbf{b}$. A1-A9 indicate dMTs in $\mathbf{d}$. Red arrowheads: sMTs with hooks, yellow arrowheads: dMTs, blue arrowheads: central tube, purple arrowheads: Y-links of the transition zone, yellow arrow: daughter centriole. $\mathbf{e}^{\prime \prime}$ Longitudinal tomographic slices showing basal bodies/axonemes. Large white arrowheads: basal bodies/axonemes, small white double arrowheads: flared dMTs at the base. $\mathbf{e}^{\prime \prime \prime}$ Quantification of SAS-6 and SAS-4 signals at the amphid sensory neuron basal bodies through embryonic development. $\mathbf{f}$ A longitudinal section of the amphid ASE neuron cilium in the adult hermaphrodite. Note flared dMTs at cilia base (arrowheads). TZ transition zone. Images in $\mathbf{b}$ are adapted by permission from Macmillan Publishers Ltd: [24]. Images in $\mathbf{c}$, $\mathbf{e}^{\prime}$ and $\mathbf{e}^{\prime \prime}$ are adapted from [25]. Images in $\mathbf{d}$ and $\mathbf{e}^{\prime \prime \prime}$ are adapted from [26]. Image in $\mathbf{f}$ is adapted from [15]. Scale bars $\mathbf{b}, \mathbf{c}, \mathbf{e}^{\prime}-\mathbf{e}^{\prime \prime \prime}, \mathbf{f} 100 \mathrm{~nm}, \mathbf{d} 50 \mathrm{~nm}$

asynchronously, such that both A-tubules with hooks and $\mathrm{AB}$ dMTs were observed within the same $70 \mathrm{~nm} \mathrm{sec-}$ tion by electron microscopy [25] (Fig. 2c). These dMTs subsequently grow asynchronously to template the transition zone and the axoneme [25]. Thus, basal bodies in amphid sensory neurons are comprised of a radially symmetric array of nine dMTs surrounding a central tube (Fig. 2d).
It is important to note, however, that since early steps in ciliogenesis have been visualized only in a subset of ciliated sensory neurons, it is unknown whether similar remodeling of centriole sMTs to dMTs accompanies basal body generation in other ciliated neuron types in $C$. elegans. For example, the transition zones of the chemosensory IL2 inner labial neurons contain 5-7 Y-linked dMTs instead of the canonical 9 dMTs $[8,15]$, raising the 
possibility that basal bodies in these neurons may exhibit distinct structural features.

\section{Basal body-associated structures}

Caenorhabditis elegans basal bodies appear to lack canonical centriole appendages as determined via transmission electron microscopy and serial section electron tomography of high pressure-frozen and freeze-substituted (HPF-FS) embryos [25, 26]. However, electrondense material of unknown identity has been observed between dMTs and the cell membrane in subciliary regions proximal to the transition zone [26]. In addition, distal appendage/transition fiber components such as DYF-19/FBF1 localize to the basal body region in adult sensory neurons [28, 29] (Fig. 1c). Since mesh-like structures in HPF-FS samples can be less clearly visible than those in chemically fixed specimens [30], these observations raise the possibility that appendage-associated proteins in C. elegans ciliated neurons may be organized in structures distinct from those observed in organisms with canonical basal bodies.

Although $C$. elegans basal bodies appear to lack canonical distal and subdistal appendages, transmission electron microscopy has shown that two distinct types of rootlets associate with basal bodies in different classes of sensory neurons. The IL1, OLQ, and BAG sensory neurons have striated rootlets, while amphid neurons contain amorphous rootlet-like material at the cilia base [8, $15,31]$. Basal bodies in amphid sensory neurons are also found in close proximity to apical junctions, cell adhesion complexes between the dendritic membrane and the membranes of surrounding glial support cells [3234]. It is possible that in the absence of transition fiberlike structures that permit docking of the basal body to the membrane, association of the basal body with the cytoskeleton and apical junctions anchors the basal body at the dendritic tip [34].

\section{Identification of basal body components}

Although no proteomic screens to identify basal body components have been performed in C. elegans, a number of genetic and functional genomics screens have led to the identification of key evolutionarily conserved components necessary for centriole assembly (e.g. [35-38]). Since only sensory neurons are ciliated in $C$. elegans, in theory, mutations in basal body genes should be readily isolated in forward genetic screens for viable animals with ciliary defects. Indeed, mutations in $d y f-19 /$ FBF1 were identified in such an unbiased screen [29]. Screens for mutants with ciliogenesis defects are not yet saturated [39], suggesting that additional basal body genes remain to be identified by this approach. $C$. elegans basal body molecules have also been identified via biochemical approaches [40] and by homology-based analyses [41].

\section{Basal body lifecycle \\ Remodeling of basal bodies}

A unique feature of $C$. elegans is that basal bodies do not appear to be present in sensory neurons in adults, leading to the notion that basal bodies degenerate in this organism [8]. However, recent studies suggest that while the central tubes of basal bodies in the amphid sensory neurons indeed degenerate during late embryonic development, the dMTs of the outer centriole wall persist throughout development $[25,26]$. This model is supported by the observations that central tube components (e.g. SAS-5, SAS-6) are lost in adult ciliated neurons [26, 42]. Interestingly, while a subset of outer centriole wall-associated proteins (e.g. HYLS-1) is retained, other components (e.g. SAS-4) are also lost in adults $[28,40]$, suggesting that the composition of the outer centriole wall is modified during development. Degeneration of the central tube, and loss of SAS-4 and SAS- 6 were noted beginning at the 1.5-fold stage of embryonic development, prior to axoneme extension $[25,26]$ (Fig. 2e' $\mathrm{e}^{\prime \prime \prime \prime}$ ). Degeneration of the central tube correlates with 'flaring' of the dMTs of the basal body [25] (Fig. 2e' $\mathrm{e}^{\prime \prime}$ ); these flared $\mathrm{dMTs}$ at the ciliary base in adults (Fig. 2f) were previously erroneously identified as transition fibers [8]. Thus, the centriole core is essential for initiation of ciliogenesis, but is dispensable for axoneme elongation.

\section{Does the basal body have the function of a centrosome?}

All ciliated cells in C. elegans are terminally differentiated sensory neurons. The vast majority of dendritic MTs in these neurons are oriented with their minus ends out (i.e. toward the dendritic tips where basal bodies are localized) $[43,44]$. In adult amphid neurons, a subset of 11-protofilament dendritic MTs is found in the periciliary membrane compartment at the cilia base $[15,33]$. These MTs occasionally enter the proximal-most few hundred nanometers of the dMT region of the remodeled basal body/axoneme; however, they do not appear to be basal body-anchored [15]. Intriguingly, $\gamma$-tubulin is present at the base of cilia in the head amphid and tail phasmid sensory neurons of adult C. elegans hermaphrodites [45]. Although the role of $\gamma$-tubulin in these classes of ciliated neurons is presently unclear, together these findings raise the possibility that basal bodies in C. elegans ciliated sensory neurons may function as microtubule organizing centers (MTOCs).

\section{Notable basal body findings and future prospects Basal body findings from C. elegans}

Although seminal studies in C. elegans led to the identification of highly conserved centriole components 
including the core centriole assembly pathway [46], basal bodies are only beginning to be studied in this organism. Recent work has delineated the series of basal body-associated events that regulate ciliary docking and import of intraflagellar transport (IFT) machinery critical for cilia assembly and maintenance. These studies have shown that the outer centriole wall/basal body component HYLS-1 recruits DYF-19/FBF1, which in turn directly interacts with the IFT-B component DYF$11 /$ IFT54 and facilitates ciliary entry of IFT particles $[26,28,29]$. In another study, the multifunctional conserved protein Girdin was identified in C. elegans as playing a major role in positioning the basal body, and shown to play a similar role in ciliated mammalian cells [34]. The recent detailed characterization of the centriole to basal body conversion and remodeling of the basal body following initiation of ciliogenesis $[25,26]$ now opens up new avenues of research into the underlying mechanisms.

\section{Strengths and future of basal body research in C. elegans} In addition to its genetic tractability and expansive research toolkit, $C$. elegans offers a number of unique advantages for cilia and basal body research. First, as indicated above, the ability to identify viable mutants defective in basal body structure and/or function allows for the identification of new basal body genes. Second, since basal body position determines the site of ciliogenesis and thus, correct cilia function, the localization of basal bodies at the dendritic tips of $C$. elegans sensory neurons provides an attractive experimental system in which to examine mechanisms of basal body positioning. Third, how the central tube is specifically targeted for degradation while largely sparing the outer centriole wall, and whether and how this degeneration is coordinated with developmental age will be exciting areas of study. The degeneration of the central tube is partly reminiscent of the process of centriole elimination observed in gametogenesis in many organisms [47], suggesting that studying this process in C. elegans may provide insights into related processes in other systems. Finally, the availability of a subset of basal body markers will now allow investigations into possibly diverse mechanisms of basal body genesis and structure in cells with unique axoneme ultrastructures. We expect that ongoing and future investigations into the biology of the nematode basal body will continue to provide new insights into the structure and function of this important organelle.

\section{Abbreviations}

MTs: microtubules; sMTs: singlet microtubules; dMTs: doublet microtubules; HPF-FS: high pressure-frozen and freeze-substituted; MTOC: microtubule organizing center.
Authors' contributions

IVN and PS researched and wrote the paper. IVN generated the figures. Both authors read and approved the final manuscript.

\section{Acknowledgements}

We apologize to authors whose work was omitted due to space and content constraints. We thank Alex Dammermann and Oliver Blacque for comments on the manuscript.

\section{Competing interests}

The authors declare that they have no competing interests.

\section{Funding}

Related work in the Sengupta Lab is funded in part by a grant from the NIH (R35 GM22463 to PS).

\section{Publisher's Note}

Springer Nature remains neutral with regard to jurisdictional claims in published maps and institutional affiliations.

Received: 9 May 2017 Accepted: 11 July 2017

Published online: 26 July 2017

\section{References}

1. Frezal L, Felix MA. C. elegans outside the Petri dish. Elife. 2015;4:05849.

2. Hodgkin J, Doniach T. Natural variation and copulatory plug formation in Caenorhabditis elegans. Genetics. 1997;146:149-64.

3. Felix MA, Duveau F. Population dynamics and habitat sharing of natural populations of Caenorhabditis elegans and C. briggsae. BMC Biol. 2012;10:59

4. Kiontke KC, Felix MA, Ailion M, Rockman MV, Braendle C, Penigault JB Fitch DH. A phylogeny and molecular barcodes for Caenorhabditis, with numerous new species from rotting fruits. BMC Evol Biol. 2011;11:339.

5. Felix MA, Braendle C. The natural history of Caenorhabditis elegans. Curr Biol. 2010;20:R965-9.

6. Sterken MG, Snoek LB, Kammenga JE, Andersen EC. The laboratory domestication of Caenorhabditis elegans. Trends Genet. 2015;31:224-31.

7. Felix MA, Braendle C, Cutter AD. A streamlined system for species diagnosis in Caenorhabditis (Nematoda: Rhabditidae) with name designations for 15 distinct biological species. PLoS ONE. 2014;9:e94723.

8. Perkins LA, Hedgecock EM, Thomson JN, Culotti JG. Mutant sensory cilia in the nematode Caenorhabditis elegans. Dev Biol. 1986;117:456-87.

9. Ward S, Thomson N, White JG, Brenner S. Electron microscopical reconstruction of the anterior sensory anatomy of the nematode Caenorhabditis elegans. J Comp Neurol. 1975;160:313-37.

10. Sulston JE, Horvitz HR. Post-embryonic cell lineages of the nematode, Caenorhabditis elegans. Dev Biol. 1977;56:110-56.

11. Sulston JE, Schierenberg E, White JG, Thomson JN. The embryonic cell lineage of the nematode Caenorhabditis elegans. Dev Biol. 1983;100:64-119.

12. Sulston JE, Albertson DG, Thomson JN. The Caenorhabditis elegans male: postembryonic development of nongonadal structures. Dev Biol. 1980;78:542-76.

13. Sherlekar AL, Lints R. Nematode Tango Milonguero-the C. elegans male's search for the hermaphrodite vulva. Semin Cell Dev Biol. 2014;33:34-41.

14. O'Hagan R, Wang J, Barr MM. Mating behavior, male sensory cilia, and polycystins in Caenorhabditis elegans. Semin Cell Dev Biol. 2014;33:25-33.

15. Doroquez DB, Berciu C, Anderson JR, Sengupta P, Nicastro D. A highresolution morphological and ultrastructural map of anterior sensory cilia and glia in C. elegans. eLife. 2014;3:e01948.

16. Silva M, Morsci N, Nguyen KC, Rizvi A, Rongo C, Hall DH, Barr MM. Cellspecific alpha-tubulin isotype regulates ciliary microtubule ultrastructure, intraflagellar transport, and extracellular vesicle biology. Curr Biol. 2017;27:968-80.

17. Silverman MA, Leroux MR. Intraflagellar transport and the generation of dynamic, structurally and functionally diverse cilia. Trends Cell Biol. 2009;19:306-16. 
18. Mukhopadhyay S, Lu Y, Qin H, Lanjuin A, Shaham S, Sengupta P. Distinct IFT mechanisms contribute to the generation of ciliary structural diversity in C. elegans. EMBO J. 2007;26:2966-80.

19. Snow JJ, OU G, Gunnarson AL, Walker MR, Zhou HM, Brust-Mascher I, Scholey JM. Two anterograde intraflagellar transport motors cooperate to build sensory cilia on C. elegans neurons. Nat Cell Biol. 2004;6:1109-13.

20. Morsci NS, Barr MM. Kinesin-3 KLP-6 regulates intraflagellar transport in male-specific cilia of Caenorhabditis elegans. Curr Biol. 2011;21:1239-44.

21. Hurd DD, Miller RM, Nunez L, Portman DS. Specific alpha- and betatubulin isotypes optimize the functions of sensory cilia in Caenorhabditis elegans. Genetics. 2010;185:883-96.

22. Brear AG, Yoon J, Wojtnyniak M, Sengupta P. Dlverse cell type-specific mechanisms localize $\mathrm{G}$ protein-coupled receptors to $C$. elegans sensory cilia. Genetics. 2014;197:667-84.

23. Sugioka K, Hamill DR, Lowry JB, McNeely ME, Enrick M, Richter AC, Kiebler LE, Priess JR, Bowerman B. Centriolar SAS-7 acts upstream of SPD-2 to regulate centriole assembly and pericentriolar material formation. eLife. 2017:6:20353.

24. Pelletier L, O'Toole E, Schwager A, Hyman AA, Muller-Reichert T. Centriole assembly in Caenorhabditis elegans. Nature. 2006;444:619-23.

25. Nechipurenko IV, Berciu C, Sengupta P, Nicastro D. Centriolar remodeling underlies basal body maturation during ciliogenesis in Caenorhabditis elegans. eLife. 2017;6:e25686.

26. Serwas D, Su TY, Roessler M, Wang S, Dammermann A. Centrioles initiate cilia assembly but are dispensable for maturation and maintenance in $C$. elegans. J Cell Biol. 2017;216:1659-71.

27. Heiman MG, Shaham S. DEX-1 and DYF-7 establish sensory dendrite length by anchoring dendritic tips during cell migration. Cell. 2009:137:344-55.

28. Wei Q, Zhang Y, Schouteden C, Zhang Y, Zhang Q, Dong J, Wonesch $\mathrm{V}$, Ling K, Dammermann A, Hu J. The hydrolethalus syndrome protein HYLS-1 regulates formation of the ciliary gate. Nat Commun. 2016;7:12437.

29. Wei Q, Xu Q, Zhang Y, Li Y, Zhang Q, Hu Z, Harris PC, Torres VE, Ling K, Hu J. Transition fibre protein FBF1 is required for the ciliary entry of assembled intraflagellar transport complexes. Nat Commun. 2013;4:2750.

30. McEwen BF, Hsieh CE, Mattheyses AL, Rieder CL. A new look at kinetochore structure in vertebrate somatic cells using high-pressure freezing and freeze substitution. Chromosoma. 1998;107:366-75.

31. Mohan S, Timbers TA, Kennedy J, Blacque OE, Leroux MR. Striated rootlet and nonfilamentous forms of rootletin maintain ciliary function. Curr Biol. 2013;23:2016-22

32. Labouesse M. Epithelial junctions and attachments. WormBook. 2006;13:1-21.

33. Kaplan OI, Doroquez DB, Cevik S, Bowie RV, Clarke L, Sanders AA, Kida K, Rappoport JZ, Sengupta P, Blacque OE. Endocytosis genes facilitate protein and membrane transport in C. elegans sensory cilia. Curr Biol. 2012;22:451-60.
34. Nechipurenko IV, Olivier-Mason A, Kazatskaya A, Kennedy J, McLachlan IG, Heiman M, Blacque OE, Sengupta P. A conserved role for Girdin in basal body positioning and ciliogenesis. Dev Cell. 2016;12:493-506.

35. Kirkham M, Muller-Reichert T, Oegema K, Grill S, Hyman AA. SAS-4 is a C. elegans centriolar protein that controls centrosome size. Cell. 2003; 112:575-87.

36. Delattre M, Leidel S, Wani K, Baumer K, Bamat J, Schnabel H, Feichtinger $\mathrm{R}$, Schnabel R, Gonczy P. Centriolar SAS-5 is required for centrosome duplication in C. elegans. Nat Cell Biol. 2004;6:656-64.

37. Leidel S, Delattre M, Cerutti L, Baumer K, Gonczy P. SAS-6 defines a protein family required for centrosome duplication in C. elegans and in human cells. Nat Cell Biol. 2005;7:115-25.

38. O'Connell KF, Caron C, Kopish KR, Hurd DD, Kemphues KJ, LiY, White JG. The C. elegans zyg-1 gene encodes a regulator of centrosome duplication with distinct maternal and paternal roles in the embryo. Cell. 2001;105:547-58.

39. Ou G, Koga M, Blacque OE, Murayama T, Ohshima Y, Schafer JC, Li C, Yoder BK, Leroux MR, Scholey JM. Sensory ciliogenesis in Caenorhabditis elegans: assignment of IFT components into distinct modules based on transport and phenotypic profiles. Mol Biol Cell. 2007;18:1554-69.

40. Dammermann A, Pemble H, Mitchell BJ, McLeod I, Yates JR 3rd, Kintner C, Desai $A B$, Oegema K. The hydrolethalus syndrome protein HYLS-1 links core centriole structure to cilia formation. Genes Dev. 2009;23:2046-59.

41. Sanders AA, de Vrieze E, Alazami AM, Alzahrani F, Malarkey EB, Sorusch N, Tebbe L, Kuhns S, van Dam TJ, Alhashem A, et al. KIAA0556 is a novel ciliary basal body component mutated in Joubert syndrome. Genome Biol. 2015:16:293.

42. Schouteden C, Serwas D, Palfy M, Dammermann A. The ciliary transition zone functions in cell adhesion but is dispensable for axoneme assembly in C. elegans. J Cell Biol. 2015;210:35-44.

43. Maniar TA, Kaplan M, Wang GJ, Shen K, Wei L, Shaw JE, Koushika SP, Bargmann CI. UNC-33 (CRMP) and ankyrin organize microtubules and localize kinesin to polarize axon-dendrite sorting. Nat Neurosci. 2011;15:48-56.

44. Hao L, Thein M, Brust-Mascher I, Civelekoglu-Scholey G, Lu Y, Acar S, Prevo B, Shaham S, Scholey JM. Intraflagellar transport delivers tubulin isotypes to sensory cilium middle and distal segments. Nat Cell Biol. 2011;13:790-8

45. Bobinnec Y, Fukuda M, Nishida E. Identification and characterization of Caenorhabditis elegans gamma-tubulin in dividing cells and differentiated tissues. J Cell Sci. 2000;113:3747-59.

46. Gonczy P. Towards a molecular architecture of centriole assembly. Nat Rev Mol Cell Biol. 2012;13:425-35.

47. Manandhar G, Schatten H, Sutovsky P. Centrosome reduction during gametogenesis and its significance. Biol Reprod. 2005;72:2-13.

\section{Submit your next manuscript to BioMed Central and we will help you at every step:}

- We accept pre-submission inquiries

- Our selector tool helps you to find the most relevant journal

- We provide round the clock customer support

- Convenient online submission

- Thorough peer review

- Inclusion in PubMed and all major indexing services

- Maximum visibility for your research

Submit your manuscript at www.biomedcentral.com/submit
BioMed Central 